\title{
artigo
}

\section{Evidências para a assistência de enfermagem à gestante com câncer de mama: revisão integrativa}

\author{
Evidence for nursing assistance to pregnant with breast cancer: an integrative review \\ Evidencia de asistencia de enfermería a embarazadas con cáncer de mama: una revisión integradora
}

\begin{abstract}
RESUMO
Objetivo: Discutir as evidências que a literatura apresenta sobre a assistência a gestante com diagnóstico de câncer de mama e as contribuições do Enfermeiro para saúde deste grupo. Método: Revisão integrativa de literatura realizada nas bases: Literatura Latino-Americana e do Caribe em Ciências da Saúde (LILACS), Literatura Internacional em Ciências da Saúde (MEDLINE) e Scientific Electronic Library Online (SciELO). Resultados: Quinze artigos compuseram a revisão. Três categorias de discussão foram criadas: 1) Câncer de mama: caracterização sociodemográfica de mulheres grávidas com neoplasia de mama; 2) Principais abordagens diagnósticas e terapêuticas frente ao câncer de mama em mulheres gravidas; 3) Assistência de Enfermagem a mulheres com diagnóstico de câncer de mama na gestação. Conclusão: 0 Enfermeiro deve ser capacitado a identificar as alterações não fisiológicas nas mamas, contribuindo na detecção precoce do câncer durante as consultas pré-natais. Apesar disto, foram encontrados poucos estudos que abordam a Assistência de Enfermagem neste contexto.
\end{abstract}

DESCRITORES: Câncer de mama; Gestantes; Assistência à saúde; Oncologia; Enfermagem.

\section{ABSTRACT}

Objective: To discuss the evidence that the literature presents on the care of pregnant women with diagnosis of breast cancer and the contributions of the Nurse to the health of this group. Method: Integrative review of literature carried out at the bases: Latin American and Caribbean Literature on Health Sciences (LILACS), International Health Sciences Literature (MEDLINE) and Scientific Electronic Library Online (SciELO). Results: Fifteen articles composed the review. Three categories of discussion were created: 1) Breast cancer: sociodemographic characterization of pregnant women with breast neoplasia; 2) Main diagnostic and therapeutic approaches to breast cancer in pregnant women; 3) Nursing assistance to women with diagnosis of breast cancer in pregnancy. Conclusion: The nurse should be trained to identify the non-physiological changes in the breasts, contributing to early detection of cancer during prenatal consultations. Despite this, few studies have been found that address Nursing Care in this context.

DESCRIPTORS: Breast cancer; Pregnant Women; Delivery of Health Care; Medical Oncology; Nursing.

\section{RESUMEN}

Objetivo: Discutir las evidencias que la literatura presenta sobre la atención a las gestantes diagnosticadas de cáncer de mama y las aportaciones de la Enfermería a la salud de este colectivo. Método: Revisión integradora de la literatura realizada en las bases: Latin American and Caribbean Health Sciences Literature (LILACS), International Health Sciences Literature (MEDLINE) y Scientific Electronic Library Online (SciELO). Resultados: Quince artículos componían la revisión. Se crearon tres categorías de discusión: 1) Cáncer de mama: caracterización sociodemográfica de las gestantes con neoplasia de mama; 2) Principales enfoques diagnósticos y terapéuticos del cáncer de mama en las gestantes; 3) Cuidados de enfermería a las mujeres diagnosticadas de cáncer de mama durante el embarazo. Conclusión: La enfermera debe estar capacitada para identificar las alteraciones no fisiológicas de las mamas, contribuyendo a la detección precoz del cáncer durante las consultas prenatales. A pesar de ello, se han encontrado pocos estudios que aborden los cuidados de enfermería en este contexto.

DESCRIPTORES: Neoplasias de la Mama; Mujeres Embarazadas; Prestación de Atención de Salud; Oncología Médica; Enfermería.

RECEBIDO EM: 29/01/2021 APROVADO EM: 12/02/2021

\section{Daiana Lima da Silva}

Enfermeira. Discente da Especialização em Enfermagem Oncológica pelo Centro Universitário Celso Lisboa. Enfermeira Supervisora da Unidade Terapia Intensiva do Hospital e Maternidade Terezinha de Jesus.

ORCID: 0000-0002-2067-1210 


\section{Giselle Simões Cotinhola de Oliveira}

Enfermeira. Discente da Especialização em Enfermagem Oncológica pelo Centro Universitário Celso Lisboa. Enfermeira Intensivista do CTI Covid do Hospital Unimed Rio.

ORCID: 0000-0003-4223-3905

\section{Patrícia Roque da Silva Pires}

Enfermeira. Especialista em Saúde da Mulher (Universidade Estácio de Sá). Discente da Especialização em Enfermagem Oncológica pelo Centro Universitário Celso Lisboa. Enfermeira da Unidade de internação do Hospital Unimed Rio.

ORCID: 0000-0001-9459-1099

\section{Raquel Vicente dos Santos Reis}

Enfermeira. Discente da Especialização em Enfermagem Oncológica pelo Centro Universitário Celso Lisboa. Enfermeira Supervisora da Unidade cardiológica do Hospital Unimed Rio.

ORCID: 0000-0002-1836-473X

\section{Regina Helena de Souza}

Enfermeira. Especialista em Saúde da Família e Comunidade. Discente da Especialização em Enfermagem Oncológica pelo Centro Universitário Celso Lisboa. Responsável técnica na CMS Cyro de Mello.

ORCID: 0000-0002-1683-884X

\section{Vanessa Moreira da Silva Soeiro}

Enfermeira. Doutoranda em Saúde Coletiva pela Universidade Federal do Maranhão (UFMA). Mestra em Enfermagem (UFMA). Especialista em Gestão em Saúde e Metodologia do Ensino Superior pela Universidade Federal do Maranhão (UFMA).

ORCID: 0000-0002-4299-1637

\section{Lucian da Silva Viana}

Enfermeiro. Doutorando em Saúde Pública e Meio Ambiente da Escola Nacional de Saúde Pública - Sergio Arouca (FIOCRUZ). Mestre em Oncologia pelo Instituto Nacional de Câncer José Alencar Gomes da Silva (INCA). Especialista em Oncologia - Residência Multiprofissional (INCA) e em Estomaterapia pela Universidade do Estado do Rio de Janeiro (UERJ). Aperfeiçoamento em Pesquisa Oncológica pelo Centro de Pesquisa (INCA). Professor convidado da Especialização em Enfermagem Oncológica do Centro Universitário Celso Lisboa.

ORCID: 0000-0002-4718-1748

\section{INTRODUÇÃO}

0 câncer de mama é a neoplasia maligna de maior incidência na população feminina. Em 2018, sua estimativa global chegava a 2,1 milhões de casos novos. No Brasil, estima-se que ocorram 66.280 casos novos de câncer de mama, para cada ano do triênio 20202022. Trata-se, portanto, de um importante problema de saúde pública ${ }^{1,2}$.

O câncer de mama diagnosticado no período gravídico-puerperal (durante a gravidez ou em um período de até 12 meses após o parto) é denominado "câncer de mama gestacional" ou "câncer de mama associado à gravidez". Apesar de ser a neoplasia maligna mais incidente em mulheres no período gravídico (principalmente entre 17 e 25 semanas), é uma situação clínica rara e desafiadora, com incidência variando de 1:3.000 a 1:10.000 gestações ${ }^{3,4}$.
As altas taxas de incidência de câncer de mama no Brasil justificam-se, dentre outros fatores, pelo diagnóstico tardio e quando a mulher se encontra no período gravídico-puerperal este diagnóstico torna-se ainda mais difícil. Isto se deve às mudanças físicas e fisiológicas que ocorrem nas mamas, próprias da gestação e que dificultam a identificação precoce de alterações mamárias ${ }^{4}$.

$\mathrm{O}$ câncer de mama na gestante vai além de questôes clínicas e condutas terapêuticas de difícil abordagem, seus impactos na saúde mental da mulher necessitam de visibilidade. Trata-se de um diagnóstico de difícil notificação para a usuária e sua família, carregado de medos e estigmas e entendido como uma sentença de morte, em um momento onde a vida seria celebrada com o nascimento de um novo membro da família, deste modo, produz sentimentos antagônicos 5 .
A Enfermagem, por acompanhar o paciente de forma mais próxima, está presente nos diferentes estágios e cenários, atuando desde a prevenção primária aos cuidados pós-diagnóstico e na reabilitação, prestando uma assistência holística. Ressalta-se ainda seu papel dentro da equipe multidisciplinar, na discussão dos casos, na tomada de decisões, na realização de planos de cuidados e na manutenção do conforto físico e emocional ${ }^{5}$.

Diante disto, este estudo tem por objetivo discutir as evidências que literatura apresenta sobre a assistência a gestante com diagnóstico de câncer de mama e as contribuições do Enfermeiro para saúde destas mulheres.

\section{MÉTODO}

Trata-se de uma revisão integrativa da literatura. Elegeu-se como questão norte- 


\section{artigo}

Lima da Silva, D.; Oliveira, G.S.C.; Pires, P.R.S.; Reis, R.V.S.; Souza, R.H.; Soeiro, V.M.S.; Viana, L.S.

Evidências para a assistência de enfermagem à gestante com câncer de mama: revisão integrativa

adora: Quais evidências a literatura apresenta sobre a assistência a gestante com diagnóstico de câncer de mama?

$\mathrm{Na}$ construção da revisão, foram seguidas as seguintes etapas: levantamento da questão de pesquisa; busca na literatura; categorização dos estudos; avaliação dos estudos incluídos na revisão; interpretação dos resultados e apresentação da síntese de conhecimento ${ }^{6}$.
Para a busca na literatura, realizada em outubro de 2020, utilizou-se o Portal Regional da Biblioteca Virtual em Saúde (BVS), através da combinação dos descritores DeSC/MeSH "Câncer de mama" e "Gravidez", por meio do operador booleano AND. As Bases de dados em saúde pesquisadas foram a Literatura Latino-Americana e do Caribe em Ciências da Saúde (LILACS), Literatura Internacional em

\section{Figura 1 - Fluxograma de busca e seleção nas bases de dados.}

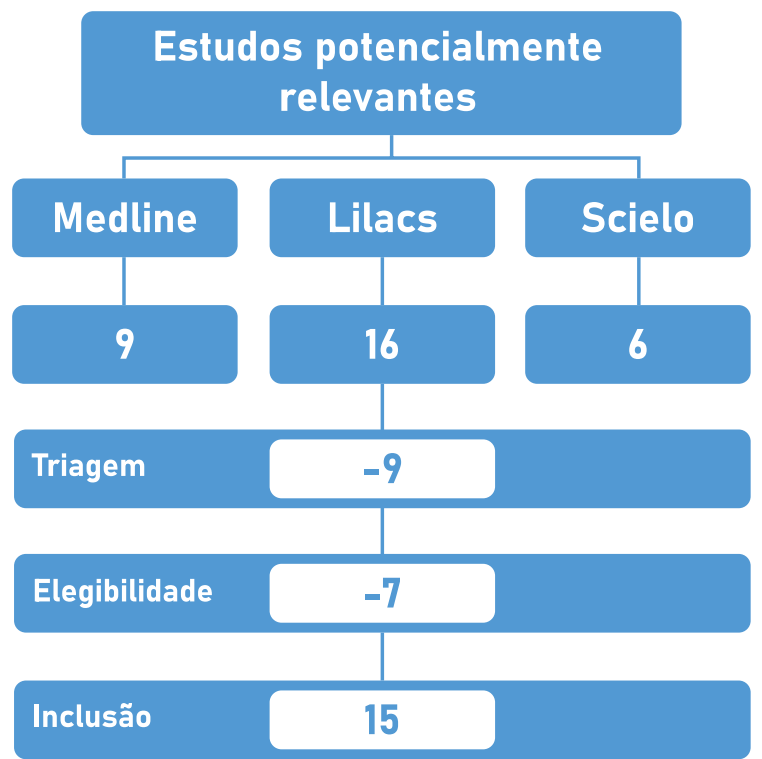

Fonte: SINAN-AIDS. 2009 - 30/06/2019

Ciências da Saúde (MEDLINE) e Scientific Electronic Library Online (SciELO).

Elegeu-se como critérios de inclusão: artigos indexados em periódicos científicos publicados entre 2010 e 2020, nos idiomas português e espanhol que abordassem a temática proposta. Foram excluídos os artigos duplicados, textos incompletos, teses, monografias, artigos de reflexão, além de publicações que não abordassem o tema.

A pré-seleção de artigos foi feita pela leitura preliminar de títulos e resumos. Os estudos pré-selecionados foram lidos na íntegra para seleção final dos artigos para análise. Após as etapas pré-definidas, 15 estudos foram selecionados para compor a revisão, conforme fluxograma da Figura 1.

Os dados dos artigos selecionados foram registrados individualmente, com destaque para autor, ano, título do artigo, metodologia.

A revisão do processo baseou-se nas recomendações da lista de conferência Preferred Reporting Items for Systematic Reviews and Meta-Analyses (PRISMA).

\section{RESULTADOS}

O resumo e caracterização dos artigos selecionados para categorização são apresentados no Quadro 1.

Observou-se que o ano com maior número de publicações foi 2015 (27\%) e a tipologia de estudos mais utilizada foi a revi-

\begin{tabular}{|c|c|c|c|c|c|}
\hline $\begin{array}{l}\text { BASE DE } \\
\text { DADOS }\end{array}$ & $\begin{array}{l}\text { PAÍS DE } \\
\text { ORIGEM }\end{array}$ & TÍTULO & TIPO DE ESTUDO & AUTOR/ANO & $\begin{array}{l}\text { PERIÓDICO E ANO DE } \\
\text { PUBLICAÇÃO }\end{array}$ \\
\hline LILACS & México & $\begin{array}{l}\text { Abordaje diagnóstico-terapéutico del } \\
\text { cáncer de mama asociado con embarazo }\end{array}$ & $\begin{array}{l}\text { Revisão } \\
\text { bibliográfica }\end{array}$ & $\begin{array}{c}\text { Gallegos-Hernán- } \\
\text { dez }\end{array}$ & $\begin{array}{l}\text { Cirugía y Cirujanos } \\
\text { (2010) }\end{array}$ \\
\hline LILACS & Brasil & $\begin{array}{l}\text { Diagnóstico de câncer de mama na } \\
\text { gestação: há dificuldades adicionais? }\end{array}$ & $\begin{array}{l}\text { Revisão } \\
\text { bibliográfica }\end{array}$ & Alquimim et al. ${ }^{8}$ & FEMINA (2011) \\
\hline SciELO & Brasil & $\begin{array}{l}\text { O prognóstico câncer de mama na } \\
\text { gravidez: evidências para o cuidado de } \\
\text { enfermagem }\end{array}$ & $\begin{array}{l}\text { Revisão } \\
\text { integrativa }\end{array}$ & Fernandes et al..$^{9}$ & $\begin{array}{l}\text { Revista Latino-Am. } \\
\text { Enfermagem (2011) }\end{array}$ \\
\hline LILACS & Brasil & Câncer de mama e gestação & $\begin{array}{l}\text { Revisão } \\
\text { bibliográfica }\end{array}$ & Martins; Lucarelli10 & FEMINA (2012) \\
\hline LILACS & Chile & $\begin{array}{l}\text { Estudio de factores obstétricos,nutri- } \\
\text { cionales y hormonales y su influencia } \\
\text { en el desarrollo de cáncer de mama }\end{array}$ & $\begin{array}{l}\text { Análise } \\
\text { retrospectiva de } \\
\text { registros médicos }\end{array}$ & $\begin{array}{l}\text { González-Jiménez; } \\
\text { Montero-Alonso; } \\
\text { Riovalle }^{11}\end{array}$ & Rev Med Chile (2013) \\
\hline
\end{tabular}




\begin{tabular}{|c|c|c|c|c|c|}
\hline SciELO & Brasil & $\begin{array}{l}\text { Câncer na gravidez e quimioterapia: } \\
\text { revisão sistemática }\end{array}$ & $\begin{array}{l}\text { Revisão } \\
\text { sistemática }\end{array}$ & Monteiro et al. ${ }^{12}$ & $\begin{array}{c}\text { Revista da Associa- } \\
\text { ção Médica Brasileira } \\
\text { (2013) }\end{array}$ \\
\hline LILACS & Brasil & Câncer de mama associado à gestação & $\begin{array}{l}\text { Revisão } \\
\text { sistemática }\end{array}$ & Ferreira; Spautz ${ }^{13}$ & FEMINA (2014) \\
\hline MEDLINE & Espanha & $\begin{array}{c}\text { Actualización del cáncer de mama en } \\
\text { atención primaria (V/V) }\end{array}$ & $\begin{array}{c}\text { Revisão } \\
\text { bibliográfica }\end{array}$ & García et al. ${ }^{14}$ & $\begin{array}{l}\text { Medicina de Familia - } \\
\text { SEMERGEN(2014) }\end{array}$ \\
\hline MEDLINE & Espanha & Consenso cáncer de mama y fertilidade & $\begin{array}{l}\text { Revisão } \\
\text { bibliográfica }\end{array}$ & Ara et al. ${ }^{15}$ & $\begin{array}{c}\text { Revista de senología } \\
\text { y patología mamaria } \\
\text { (2015) }\end{array}$ \\
\hline LILACS & Brasil & $\begin{array}{l}\text { Caracterização e capacidade funcional } \\
\text { de mulheres com câncer ginecológico, } \\
\text { câncer mamário e doença trofoblástica }\end{array}$ & $\begin{array}{l}\text { Estudo } \\
\text { longitudinal } \\
\text { retrospectivo }\end{array}$ & Elias et al. ${ }^{16}$ & $\begin{array}{l}\text { Revista Gaúcha de } \\
\text { Enfermagem (2015) }\end{array}$ \\
\hline MEDLINE & Espanha & $\begin{array}{c}\text { Biopsia selectiva de ganglio centinela } \\
\text { en mujer embarazada con cáncer de } \\
\text { mama }\end{array}$ & Relato de caso & $\begin{array}{l}\text { Fernandez; Zamo- } \\
\text { ra; Marcos }{ }^{17}\end{array}$ & $\begin{array}{c}\text { Progresos de Obs- } \\
\text { tetricia y Ginecología } \\
\text { (2015) } \\
\end{array}$ \\
\hline MEDLINE & Espanha & $\begin{array}{c}\text { Influencia de los factores reproducti- } \\
\text { vos, la lactancia materna y la obesidad } \\
\text { sobre el riesgo de cáncer de mama en } \\
\text { mujeres mexicanas }\end{array}$ & $\begin{array}{l}\text { Estudo de caso e } \\
\text { controle }\end{array}$ & $\begin{array}{l}\text { Navarro-Ibarra } \\
\text { et al. }{ }^{18}\end{array}$ & $\begin{array}{l}\text { Nutrición Hospitalaria } \\
\text { (2015) }\end{array}$ \\
\hline MEDLINE & Espanha & $\begin{array}{c}\text { Tratamiento Neoayuvante en el cáncer } \\
\text { de mama gestacional }\end{array}$ & $\begin{array}{l}\text { Revisão } \\
\text { bibliográfica e } \\
\text { relato de caso }\end{array}$ & Sánchez et al. ${ }^{19}$ & $\begin{array}{c}\text { Progresos de Obs- } \\
\text { tetricia y Ginecología } \\
\text { (2017) } \\
\end{array}$ \\
\hline LILACS & Brasil & $\begin{array}{l}\text { Câncer de mama na gestação: aborda- } \\
\text { gem diagnóstica e terapêutica. }\end{array}$ & $\begin{array}{l}\text { Revisão } \\
\text { bibliográfica }\end{array}$ & Silva et al..$^{20}$ & $\begin{array}{l}\text { Acta médica - Porto } \\
\text { Alegre (2018) }\end{array}$ \\
\hline LILACS & Brasil & $\begin{array}{l}\text { Fatores associados ao câncer de mama } \\
\text { gestacional: estudo caso-controle }\end{array}$ & $\begin{array}{c}\text { Estudo } \\
\text { observacional }\end{array}$ & Monteiro et al..$^{21}$ & $\begin{array}{l}\text { Ciência \& Saúde } \\
\text { Coletiva (2019) }\end{array}$ \\
\hline
\end{tabular}

são bibliográfica (47\%). Os estudos foram agrupados em categorias temáticas para ampliar as discussões, a saber (Quadro 2):

\section{DISCUSSÃO}

Câncer de mama: caracterização sociodemográfica de mulheres grávidas com neoplasia de mama
De acordo com literatura, o câncer de mama gestacional (CMG) possui taxa de incidência compreendida aproximadamente entre 1 a 3 por 10 mil, sendo o tipo de câncer mais comum durante a gestação ${ }^{7,13,17}$. Esta frequência pode aumentar com a idade, sendo maior em mulheres acima de 35 anos. O adiamento da primeira gestação é o aspecto social mais mencio- nado nos estudos, pois esta tende a coincidir com as morbidades características da idade $\mathrm{i}^{12,13,17,20}$.

Por se tratar de uma característica sociocultural, a faixa etária pode variar de acordo com o país. No México as mulheres entre 24 a 44 anos são as mais afetadas 7 . Já em estudo realizado em Uberaba (MG), a prevalência foi maior entre mulheres

\section{Quadro 2 - Categorização dos estudos.}

\section{TÍTULO DA CATEGORIA}

Câncer de mama: caracterização sociodemográfica de mulheres grávidas com neoplasia de mama

Principais abordagens diagnósticas e terapêuticas frente ao câncer de mama em mulheres gravidas.

Assistência de Enfermagem a mulheres com diagnóstico de câncer de mama na gestação

\section{AUTORES}

Gallegos-Hernández (2010); Montero-Alonso; Riovalle (2013); Monteiro et al (2013); Ferreira; Spautz (2014); Fernandez; Zamora; Marcos (2015); NavarroIbarra et al. (2015); Elias et al. (2015); González-Jiménez; Ara et al (2015); Silva et al (2018); Monteiro et al (2019);

Gallegos-Hernández (2010); Alquimim et al. (2011); Martins; Lucarelli (2012); Monteiro et al. (2013); Ferreira; Spautz (2014); Fernandez; Zamora; Marcos (2015); Sanchez et al. (2017); Silva et al (2018);

Fernandes et al. (2011); Vieira et al. (2012); García et al. (2014). 
com 41 e 50 anos $^{16}$. Com relação ao grau de instrução, diferentes estudos concordam que o CMG prevalece em mulheres de menores escolaridades e níveis socioeconômicos ${ }^{16,18,21}$.

O número de filhos também demonstrou ser influente, o risco de desenvolver câncer de mama em mulheres que engravidaram após os 35 anos é maior do que em nulíparas da mesma faixa etária e duas vezes superior ao de mulheres que engravidaram com menos de 20 anos. Mulheres com 1 filho são duas vezes mais suscetíveis ao câncer de mama que as com 4 ou 5 filhos e o intervalo de até 2 anos entre as gravidezes contribui para a diminuição da incidência de câncer de mama em gestações até 30 anos $^{11,21}$.

Há estudos que indicam que para usuárias jovens de anticoncepcional, há um aumento discreto no risco, que diminui após a interrupção do uso e após 10 anos da descontinuação, enquanto outros não evidenciaram relação entre a neoplasia e o contraceptivo ${ }^{14,17}$. Quanto aos hábitos alimentares, foi constatado que sobrepeso e obesidade favorecem alterações nos níveis de prolactina e estrogênio, logo pode se caracterizar como fator de risco visto que o câncer tem relação direta com esses hormônios ${ }^{10}$. Identificar o perfil e hábitos da gestante auxilia no diagnóstico precoce ainda na unidade básica de saúde, visto que a Atenção Primária é o primeiro atendimento das usuárias no Sistema Único de Saúde (SUS).

É válido salientar que além desses fatores de risco supracitados, outros referentes a comportamentos ou ao ambiente, como a exposição à radiação ionizante e tabagismo já estão fortemente estabelecidos na literatura nacional e internacional. Assim como fatores comportamentais, chamados de fatores de proteção, que ajudam a diminuir o risco desse tipo de câncer, como a amamentação e prática de atividade física ${ }^{2,22}$.

\section{Principais abordagens diagnósticas e terapêuticas frente ao câncer de mama em mulheres gravidas}

A literatura define como CMG aquele que tem seu diagnóstico ocorrido durante

a gestação ou até um ano após o parto. Em virtude de os exames de rastreio de neoplasias mamárias não fazerem parte das rotinas de pré-natal, seu diagnóstico pode ser tardio. Além disto, as alterações mamárias que ocorrem de modo fisiológico durante o ciclo gravídico (aumento do volume mamário, da vascularização e da retenção hídrica), dificultam a identificação de alterações durante o exame clínico das mamas e nas mamografias. Deste modo, indica-se que a realização da mamografia seja feita no período pré-gestacional, em especial em mulheres com histórico familiar de neoplasias de mama ${ }^{8,10}$.

É importante

destacar que a

realização do

pré-natal

pode ser uma

oportunidade única

de identificação

oportuna do

câncer de mama

dado ao contínuo

contato entre

usuária gestante

e profissional de

saúde.
A biópsia é a única maneira de descobrir se uma alteração mamária é câncer. É realizada técnica de punção na mama afetada, utilizando uma agulha fina, em ambiente ambulatorial, sob anestesia local. Porém, em alguns casos é necessária uma biópsia cirúrgica, que requer anestesia geral, e implica pequeno risco para o feto ${ }^{23}$. Sobre a biópsia de linfonodo sentinela durante a gravidez ainda não há consenso sobre sua efetividade, visto que o sistema linfático também sofre alterações devido ao processo gestacional. Esta técnica está associada a materiais radioativos, mas em níveis não teratogênicos, sendo necessária a realização de mais estudos sobre sua segurança ${ }^{10,13}$.

A literatura indica que a tomografia computadorizada e a cintilografia óssea têm seu uso limitado durante a gestação, tendo em vista a exposição fetal a radiação. Quando na possibilidade de metástases ósseas, recomenda-se uso com limitação de doses de radiação ou a ressonância sem contraste, visto que este pode afetar o feto ${ }^{7,20,23}$. Existe relutância dos profissionais em solicitar exames radiográficos e invasivos no período gestacional, contribuindo para diagnósticos tardios. Embora ainda se discuta muito sobre a eficácia da mamografia em mulheres grávidas, a literatura aponta que não há contraindicação na sua execução durante a gravidez, por ter liberação considerada baixa de radiação, desde que haja adequado uso de equipamento de proteção abdominal. ${ }^{7.13}$. Já os exames de ultrassom da mama não usam radiação e são considerados seguros durante a gravidez. Por ser um equipamento mais acessível e de fácil utilização no nível de atenção primária, geralmente é o primeiro teste feito para avaliar uma alteração na mama ${ }^{23}$.

Dentre outros motivos que podem atrasar o diagnóstico a literatura destaca a confusão de nódulos com mastite e a resistência da paciente à biópsia. É importante destacar que a realização do pré-natal pode ser uma oportunidade única de identificação oportuna do câncer de mama dado ao contínuo contato entre usuária gestante e profissional de saúde. Faz-se necessário 
otimizar este espaço como facilitador do diagnóstico ${ }^{7,8,10}$.

Em casos confirmados de câncer de mama durante a gestação, a indicação cirúrgica segue as mesmas etapas recomendadas em mulheres não grávidas ${ }^{10,13,20}$. Cirurgias conservadoras necessitam de radioterapia complementar, e neste caso, o procedimento é teratogênico, sendo excluído. A quimioterapia deverá ser a opção mais eficaz nestes casos. Para carcinomas em estágio I, a literatura indica abordagem cirúrgica, podendo ser realizado no final do terceiro trimestre por mastectomia ${ }^{10}$.

Já, sobre a anestesia, os efeitos colaterais são mínimos para o feto, ocorrendo em casos raros, comprometimento do crescimento fetal, aborto e prematuridade. No organismo materno, as alterações fisiológicas relacionadas à gestação - cardiovasculares, pulmonares, diafragmáticas e gástricas - podem complicar o quadro durante a anestesia geral ${ }^{10}$.

Alguns autores ${ }^{13}$ afirmam que a cirurgia das mamas pode ser seguramente realizada em qualquer trimestre da gestação com risco mínimo para o desenvolvimento fetal, de técnicas mais conservadoras a mastectomias, sendo essa última considerada "padrão-ouro". Outros, entretanto, indicam que o momento mais seguro para a realização da cirurgia é no segundo trimestre, uma vez que no terceiro trimestre há risco aumentado de parto prematuro devido ao estresse da cirurgia ${ }^{12,20}$. Quanto a cirurgia de reconstrução mamária, os estudos selecionados concordam como melhor opção a realização desta após o parto ${ }^{10,13}$.

Em relação à quimioterapia, esta pode ser utilizada em até 12 semanas da cirurgia de mama, sendo contraindicada nas quatro semanas antes do parto, no primeiro trimestre gestacional e durante a lactação. Seu uso deve ser discutido individualmente ${ }^{10,12,17}$.

É importante destacar que não há casos relatados de câncer de mama com efeito direto sobre o feto, contudo, em casos raros, o câncer pode atinge a placenta, alterando o seu estado nutricional. Além desse risco sobre o feto, o uso de medicamentos citotóxicos na gestação está relacionado a maior risco de má formação do feto, principalmente no primeiro trimestre gestacional. Seu uso pode ser realizado com maior segurança no segundo trimestre, porém há relatos na literatura de associação com óbitos fetais e restrição de crescimento, além disto, a efetividade do quimioterápico é discutível, uma vez que alterações fisiológicas maternas (hormonais e hepáticas) podem diminuir a concentração plasmática da droga no organismo materno, interferindo em sua eficácia ${ }^{13,19}$.

\section{Em relação à}

quimioterapia, esta

pode ser utilizada

em até 12 semanas

da cirurgia de

mama, sendo

contraindicada nas

quatro semanas

antes do parto, no

primeiro trimestre

gestacional e

durante a lactação.

Seu uso deve

ser discutido

individualmente.
Assistência de enfermagem a muIheres com diagnóstico de câncer de mama na gestação

O Enfermeiro está intimamente relacionado ao bom prognóstico do câncer de mama na gravidez. Durante as consultas de pré-natal e puerpério faz-se necessário a realização do exame clínico das mamas, sendo o enfermeiro profissional capacitado para identificação de alterações não fisiológicas e achados anormais nas mamas, contribuindo na detecção precoce do câncer. Contudo, a literatura aponta que ainda há maior preocupação com a saúde fetal em detrimento da materna durante as consultas de pré-natal, refletindo um modelo biologista ainda hegemônico. É necessário romper com estas práticas, fortalecendo a humanização da assistência e oferecendo um cuidado que considere o ambiente social, econômico, cultural e físico a que pertence à usuária, bem como a participação da família junto aos processos decisórios?

Como competências do Enfermeiro na assistência a gestante com câncer de mama a literatura aponta: a atenção integral; avaliação contínua e individualizada no pré-natal; ações de prevenção e detecção precoce do câncer de mama em todos os níveis de atenção; preparo profissional para exame clínico das mamas; e ações educativas com a população?.

A abordagem no câncer de mama durante o período gravídico requer um caráter biopsicossocial em sua condução. A literatura aponta que estas usuárias estão susceptíveis a níveis de estresse mais elevados, podendo ocorrer depressão e ansiedade, principalmente no primeiro ano de diagnóstico. As alterações que ocorrem no corpo devido à patogenia do câncer, somadas as alterações fisiológicas da gestação, afetam a autoimagem e sexualidade da gestante. Como recursos terapêuticos sugeridos para estes casos encontram-se a terapia cognitivo comportamental, a prática de atividade física, e a implementação de técnicas de educação sexual baseadas no conhecimento do próprio corpo, como por exemplo, as oficinas de educação sexual realizadas pelo enfermeiro ${ }^{9,14}$.

Neste contexto, a prática da Assistência 


\section{artigo}

Lima da Silva, D.; Oliveira, G.S.C.; Pires, P.R.S.; Reis, R.V.S.; Souza, R.H.; Soeiro, V.M.S.; Viana, L.S.

Evidências para a assistência de enfermagem à gestante com câncer de mama: revisão integrativa

de Enfermagem traz uma oportunidade única de escuta e acolhimento, possibilitando a identificação de sinais de alerta, bem como a orientação de cuidados e práticas educativas. Através de uma abordagem psicossocial, o Enfermeiro como integrante de uma equipe multidisciplinar, também pode contribuir para construção de projetos terapêuticos singulares que visem a melhor qualidade de vida da mulher gestante com diagnóstico de câncer de mama.

Ainda são poucos os estudos que abordam a Assistência de Enfermagem neste contexto, havendo a necessidade de condução de estudos direcionados para a atuação da equipe de Enfermagem, promovendo assim, melhoria do cuidado no contexto da atenção à saúde ${ }^{9}$.

\section{CONCLUSÃO}

Sendo uma das competências do enfermeiro a realização do pré-natal na Atenção Primária em Saúde, este profissional deve ser capacitado a identificar as alterações não fisiológicas e achados anormais nas mamas, contribuindo na detecção precoce do câncer durante as consultas, uma vez que o bom prognóstico está relacionado ao diagnóstico oportuno por meio da identificação de alertas e orientação dos cuidados. Deste modo, a atuação do profissional de enfermagem contribui significativamente para o enfrentamento do câncer como problema de saúde pública. Apesar da contribuição ímpar esta categoria pode fornecer, poucos estudos tem se debruçado a compreender a Assistência de Enfermagem neste contexto. -

\section{REFERÊNCIAS}

1. Bray F. et al. Global cancer statistics 2018: GLOBOCAN estimates of incidence and mortality worldwide for 36 cancers in 185 countries. CA: a cancer journal for clinicians, Hoboken, 2018;68(6):394-424.

2. Instituto Nacional de Câncer José Alencar Gomes da Silva (INCA). Estimativa 2020: incidência de câncer no Brasil. Rio de Janeiro: INCA, 2019.

3. Lima BC, Carvalho JÁ, Oliveira MP, Gomes JBM. Câncer de mama na gestação: um relato de caso. E- Sciencia, 2019;12(2):18-21.

4. Monteiro DL, Menezes DC, Nunes CL, Antunes CA, Almeida EM, Trajano AJ. Câncer de mama na gravidez: diagnóstico e tratamento. Revista Hospital Universitário Pedro Ernesto, 2014; 13(3):67-71.

5. Costa AELD, Souza JR. Implicações psicossociais relacionadas à assistência à gestante com câncer: percepções da equipe de saúde. Revista da SBPH, 2018;21(2):100-122.

6. Mendes KS, Silveira RCCP, Galvão CM. Revisão integrativa: método de pesquisa para a incorporação de evidências na saúde e na enfermagem. Texto \& contexto enfermagem, 2008;17(4): 758-764.

7. Gallegos-Hernández JF. Abordaje diagnóstico-terapéutico del cáncer de mama asociado con embarazo. Actualización 2010. Cir Cir 2010;78:273-282.

8. Alquimim AF, Ladeia LSA, Rodrigues RK, Oliveira VB, Éscobar EGVF, Menezzi PTSD. Diagnóstico de câncer de mama na gestação: há dificuldades adicionais? FEMINA, 2011;39(5):281-284.

9. Fernandes AFC, Santos MCL, Castro TB, Galvão CM. O prognóstico de câncer de mama na gravidez: evidências para o cuidado de enfermagem. Revista Latino-Americana de Enfermagem, 2011;19(6):[10 telas].

10. Martins MM, Lucarelli AP. Câncer de mama e gestação. FEMINA, 2012;40(4):203-207.

11. González-Jiménez E, Montero-Alonso MA, Riovalle JA. Estudio de factores obstétricos, nutricionales y hormonales y su influencia en el desarrollo de cáncer de mama. Rev Med Chile 2013;141:1541-1546.

12. Monteiro DLM et al. Câncer de mama na gravidez e quimiotera- pia: revisão sistemática. Rev assoc med bras. 2013; 59(2):174-180.

13. Ferreira LRG, Spautz CC. Câncer de mama associado à gestação. FEMINA, 2014;42(4):203-208.

14. García ND et al. Actualización del cáncer de mama en atención primaria (V/V). Semergen. 2015;41(2):76-88.

15. Ara C. et al. Consenso Cáncer de Mama y Fertilidad. Rev Senol Patol Mamar. 2015;28(4):172-180.

16. Elias TC et al. Caracterização e capacidade funcional de mulheres com câncer ginecológico, câncer mamário e doença trofoblástica gestacional. Rev Gaúcha Enferm. 2015;36(4):37-42.

17. Fernandez RA, Zamora CPF, Marcos MCL. Biopsia selectiva de ganglio centinela en mujer embarazada con cáncer de mama. Prog Obstet Ginecol. 2015;58(6):283-286.

18. Navarro-Ibarra MJ et al. Influencia de los factores reproductivos, la lactancia materna y la obesidad sobre el riesgo de cáncer de mama en mujeres mexicanas. Nutr Hosp. 2015;32(1):291-298.

19. Sánchez MCG, Díaz CMM, Gallardo MB, Ageitos AG, Antón MAH, Díaz ES. Tratamiento neoayuvante en el cáncer de mama gestacional. Progresos de obstetricia y ginecología: revista oficial de la Sociedad Española de Ginecología y Obstetricia, 2017; 60(6):594-596.

20. Silva KM, Rockenbach BF, Moura JE, Souza ABA. Câncer de mama na gestação: abordagem diagnóstica e terapêutica. Acta Medica, 2018;39(2):61-69.

21. Monteiro DLM, Nunes CL, Rodrigues NCP, Antunes CA, Almeida EM, Barmpas DBS, Magalhães ALC, Trajano AJB. Fatores associados ao câncer de mama gestacional: estudo caso-controle. Ciênc. saúde coletiva [Internet]. 2019; 24(6): 2361-2369.

22. World Cancer Research Fund; American Institute For Cancer Research. Continuous update project report: food, nutrition, physical activity, and the prevention of breast cancer. Washington, DC: AICR, 2018.

23. American Cancer Society. Finding Breast Cancer During Pregnancy. 2019. Disponivel em: https://www.cancer.org/cancer/breast-cancer/screening-tests-and-early-detection/finding-breast-cancer-during-pregnancy.html. Acesso em 18 jan. 2021. 